\title{
Wave transport in random media: The ballistic to diffusive transition
}

\author{
Z. Q. Zhang, ${ }^{1}$ I. P. Jones, ${ }^{2, *}$ H. P. Schriemer, ${ }^{2, \dagger}$ J. H. Page, ${ }^{2}$ D. A. Weitz, ${ }^{3}$ and Ping Sheng ${ }^{1}$ \\ ${ }^{1}$ Department of Physics, The Hong Kong University of Science and Technology, Clear Water Bay, Kowloon, Hong Kong \\ ${ }^{2}$ Department of Physics, University of Manitoba, Winnipeg, Manitoba, Canada R3T 2N2 \\ ${ }^{3}$ Department of Physics and Astronomy, University of Pennsylvania, Philadelphia, Pennsylvania 19104-6396
}

(Manuscript received 31 March 1999)

\begin{abstract}
The character of wave transport through a strongly scattering medium, excited by a pulsed plane-wave source, is investigated as a function of sample thickness over the range from about one to 13 mean free paths. To examine the behavior theoretically, we perform a first-principles calculation of both the frequency correlation function of the transmitted field and the time-domain profile of the transmitted intensity. These quantities are investigated experimentally using an ultrasonic technique, which allows us to separate the ballistic and scattered components of the total transmitted field, and hence to measure the scattered component unambiguously in thin samples. For sample thicknesses greater than about four mean free paths, we find good agreement between our theory, the diffusion approximation, and our experimental data for both the frequency correlation function and the intensity time profile. In thinner samples, there are systematic differences between theory and experiment. To characterize the transition from ballistic to diffusive behavior in thin samples, we focus on the arrival time of the peak in the scattered component of the transmitted intensity; unexpectedly we find that the scattered peak arrival time exhibits an abrupt crossover between ballistic and diffusive behavior when the ratio of sample thickness to mean free path, $L / \ell$, is approximately equal to 3 . Excellent agreement is obtained between our theory and experiment for this crossover behavior over the entire range of sample thicknesses investigated. [S1063-651X(99)12009-9]
\end{abstract}

PACS number(s): 42.25.Dd, 05.60. $-\mathrm{k}, 43.35 .+\mathrm{d}$

\section{INTRODUCTION}

Wave transport is known to acquire diffusive character in the presence of multiple scattering [1]; as a result, the diffusion approximation has been used very successfully to describe a wide range of fascinating wave phenomena in strongly scattering materials, including coherent backscattering, continuous-wave transmission, pulse propagation, and frequency, spatial, and temporal correlations [2-8]. Despite the success and widespread used of this simple approximation, limitations exist. One such limitation must occur in thin samples, where the number of scatterings becomes insufficient to randomize the phases of the emerging waves, and a crossover to ballistic transport must ultimately occur. However, the nature of the ballistic to diffusive transition is not fully understood, despite various approaches that have been used to study this crossover. Many of these investigations have probed the sample thickness at which the diffusion approximation breaks down, and have found differing answers to this question depending on the nature of the experiment and the degree to which deviations from diffusion theory are considered to be significant. For example, diffusing wave spectroscopy (DWS) experiments [9] and steady state photon transmission measurements $[4,10]$ have indicated that the transport of photons is diffusive for sample thicknesses, $L$, as low as $3-5$ transport mean free paths, $\ell^{*}$. In contrast, pulsed

\footnotetext{
*Present address: National Center for Physical Acoustics, Coliseum Drive, University, MS 38677.

†Present address: Van der Waals-Zeeman Instituut, Universiteit van Amsterdam, Valckenierstraat 65, 1018 XE, Amsterdam, The Netherlands.
}

optical transmission measurements [10-12] have reported systematic deviations from diffusion theory at much larger values of $L / \ell^{*} \leqslant 8-10$. In investigating these departures from diffusive behavior, the importance of correctly describing the sample boundary conditions within the diffusion approximation has also been emphasized $[4,10]$. Other investigations have considered ways of extending the diffusion approximation to describe wave transport in thinner samples. Within the context of DWS, a solution of the transport equation has been derived to account for the contributions of short scattering paths, which are increasingly ballistic in nature, giving a simple correction to the diffusion approximation that gives better agreement with experiment [13]. In another approach, the telegrapher equation with suitable boundary conditions has been employed to account for the role of ballistic transport and scattering anisotropy, thus extending the range of sample thicknesses over which DWS can be used down to about two transport mean free paths [14]. Recently, another way of studying the crossover from ballistic to diffusive behavior has been demonstrated in microwave experiments, where the statistical characteristics of the amplitude and phase of the transmitted field have been directly investigated [15]. These experiments were able to probe the extent to which the phases of continuous waves are randomized by scattering as the sample thickness and number of excited modes are varied. Despite the considerable progress that has been achieved in characterizing this crossover, puzzles remain in seeking a quantitative understanding wave transport in thin strongly scattering samples [10], motivating new theoretical and experimental approaches to explore this behavior. While these issues are of considerable scientific interest, they are also of great practical importance, as an understanding of the transition from ballistic to diffu- 
sive behavior is essential for many applications that rely on multiply scattered waves, such as optical imaging of tissue and other biological materials [16].

In this paper, we combine a first-principles theoretical calculation with detailed ultrasonic measurements to explore the nature of the transition from ballistic to diffusive behavior in thin samples of a strongly scattering material. We consider a slab geometry in which a plane-wave pulse is incident on one side of the sample, and calculate both the temporal distribution and the frequency correlation function of the transmitted waves that reach the opposite face of the sample. One advantage of focusing on pulse propagation is that we are able to examine contributions to wave transport through the medium of scattering paths of different lengths. We go beyond the limitations of the diffusion approximation by solving the Bethe-Salpeter equation numerically in the spacefrequency domain to obtain the field-field frequency correlation function of the transmitted waves, allowing us to calculate the contributions of both the ballistic and scattered components of the total wave field. These solutions should apply to samples of any thickness, and are shown to correspond to those obtained using the diffusion approximation for thick samples. Our calculations of the frequency correlation function are also the basis for determining the temporal profile of the transmitted intensity, which is obtained by taking the Fourier transform of the frequency correlation function.

We also investigate wave transport experimentally using a pulsed ultrasonic technique, thereby exploiting the phase sensitivity of piezoelectric detectors to measure the full transmitted wave field, and allowing us to separate the coherent ballistic component from the incoherent scattered component. Since previous studies of the ballistic to diffusive transition have been based on optical and microwave experiments, the use of ultrasonic techniques provides a complementary experimental approach to gaining information on the crossover behavior, not only because of differences in how ultrasonic waves are generated and detected but also because of differences in the physics of the scattering, including the relative importance of scattering anisotropy. We compare our experimental data and first-principles calculations with diffusion theory for both the ensembleaveraged frequency correlation function of the transmitted field and the time profile of the transmitted intensity; this comparison shows that, for a plane-wave input pulse, diffusive behavior is observed when the sample thickness is greater than about four transport mean free paths. The character of wave transport as a function of sample thickness is investigated by focusing on the dominant path length of the scattered waves, corresponding to the peak arrival time of the transmitted intensity. Remarkably, we find both theoretically and experimentally that the peak transmission time shows a sharp demarcation between ballistic behavior and diffusive behavior when the medium is approximately three mean free paths thick. This sharp demarcation point in the peak arrival time also corresponds quite closely to the thickness below which we find significant deviations from diffusion theory in the entire time profile of the transmitted intensity and in the frequency correlation function of the transmitted scattered field. The peak arrival time of the scattered waves provides a useful criterion for describing the transition from ballistic to diffusive behavior as a function of sample thickness, since it is relatively insensitive to the complicating effects of dispersion, scattering anisotropy, boundary reflections, and local fluctuations in the number density of scatterers.

The organization of the paper is as follows. In Sec. II, we summarize our theoretical approach, a preliminary account of which has been given elsewhere [17]. Here we focus on the underlying assumptions of the method, and give the equations that must be solved numerically to obtain the response of the medium to an incident pulse. The ultrasonic experiments that are used to investigate the predictions of this model are described in Sec. III. Section IV presents and compares our theoretical and experimental results for wave transport as a function of sample thickness, highlighting results that have not been appreciated in previous studies of the ballistic to diffusive transition. The paper ends with a summary of our main conclusions.

\section{THEORY}

Consider a plane-wave pulse that is normally incident on the front surface, $z=0$, of a slab-shaped sample containing random scatterers. The thickness of the sample is $L$. To investigate wave transport through this scattering medium, we are interested in modeling the temporal evolution of the intensity and the frequency correlation function of the field, measured at the back face of the sample at $z=L$. At any time $t$ and position $\vec{r}$, the field is specified by the time-dependent wave function, which can be written as

$$
\psi(t, \vec{r})=(2 \pi)^{-1} \int d \Omega \exp (-i \Omega t) f(\Omega) \phi_{\Omega}(\vec{r}),
$$

where $f(\Omega)$ describes the spectrum of frequencies $\Omega$ contained in the pulse, and $\phi_{\Omega}(\vec{r})$ is the spatial part of the wave function. For the incident plane wave, $\phi_{\Omega}(\vec{r})$ has the form $\exp \left(i \Omega_{z} / \mathrm{V}\right)$, where $\mathrm{v}$ is the phase velocity. Inside the sample, $\phi_{\Omega}(\vec{r})$ is determined by the superposition of the ballistic wave and all the scattered waves at position $\vec{r}$; thus it reflects details about the positions of all the scatterers which are too sample specific to convey useful information. It is therefore essential to examine configurationally averaged quantities. The ensemble-averaged field describes only the ballistic contribution, since the scattered fields cancel out as a result of configurational averaging. To include the scattered waves, the relevant quantity is the ensemble-averaged intensity, which is proportional to

$$
\begin{aligned}
\left\langle|\psi(t, \vec{r})|^{2}\right\rangle= & (2 \pi)^{-2} \int d \Omega\left[\int d \omega f\left(\Omega^{+}\right) f^{*}\left(\Omega^{-}\right)\right. \\
& \left.\times\left\langle\phi_{\Omega^{+}}(\vec{r}) \phi_{\Omega^{-}}^{*}(\vec{r})\right\rangle \exp (-i \omega t)\right],
\end{aligned}
$$

where \langle\rangle denotes configurational averaging, and the frequencies $\Omega^{ \pm}=\Omega \pm(\omega / 2)$ have been written in terms of the center frequency $\Omega$ and the difference or modulation frequency $\omega$, the latter also being conjugate to the travel time $t$. From Eq. (2) it is clear that the ensemble-averaged intensity $\left\langle|\psi(t, \vec{r})|^{2}\right\rangle$ is given by the Fourier transform of the frequency correlation function $C_{\Omega}(\omega, \vec{r})=\left\langle\phi_{\Omega^{+}}(\vec{r}) \phi_{\Omega^{-}}^{*}(\vec{r})\right\rangle$, which is the fundamental quantity to be determined. 
$C_{\Omega}(\omega, \vec{r})$ can be obtained from the space-frequency correlation function $\widetilde{C}_{\Omega}\left(\omega ; \vec{r}, \vec{r}^{\prime}\right)=\left\langle\phi_{\Omega^{+}}(\vec{r}) \phi_{\Omega^{-}}^{*}\left(\vec{r}^{\prime}\right)\right\rangle$, which is known to satisfy the Bethe-Salpeter equation

$$
\begin{aligned}
\widetilde{C}_{\Omega}\left(\omega ; \vec{r}, \vec{r}^{\prime}\right)= & \left\langle\phi_{\Omega^{+}}(\vec{r})\right\rangle\left\langle\phi_{\Omega^{-}}^{*}\left(\vec{r}^{\prime}\right)\right\rangle \\
& +\int d \vec{r}_{1} d \vec{r}_{2} d \vec{r}_{3} d \vec{r}_{4}\left\langle G_{\Omega^{+}}\left(\vec{r}, \vec{r}_{1}\right)\right\rangle \\
& \times\left\langle G_{\Omega^{-}}^{*}\left(\vec{r}^{\prime}, \vec{r}_{2}\right)\right\rangle U\left(\vec{r}_{1}, \vec{r}_{3} ; \vec{r}_{2}, \vec{r}_{4}\right) \\
& \times \widetilde{C}_{\Omega}\left(\omega ; \vec{r}_{3}, \vec{r}_{4}\right),
\end{aligned}
$$

where $\left\langle\phi_{\Omega}(\vec{r})\right\rangle$ is the configurationally averaged wave function inside the sample, and $\langle G\rangle$ and $U$ are, respectively, the ensemble-averaged Green's function and the irreducible vertex function. Since for our purpose only the frequency correlation function $C_{\Omega}(\omega, \vec{r})$ is needed, we can solve the Bethe-Salpeter equation for the simpler case where $\vec{r}^{\prime}=\vec{r}$. To carry out the calculation, we note that the configurationally averaged Green's function $\left\langle G_{\Omega}\left(\vec{r}, \vec{r}_{1}\right)\right\rangle$, which describes the coherent part of wave propagation from $\vec{r}_{1}$ to the observation point $\vec{r}$, has the approximate form [18]

$$
\left\langle G_{\Omega}\left(\vec{r}, \vec{r}_{1}\right)\right\rangle \approx-\frac{\exp \left(i K\left|\vec{r}-\vec{r}_{1}\right|\right)}{4 \pi\left|\vec{r}-\vec{r}_{1}\right|}
$$

and is assumed to be translationally invariant inside the sample. Here $K=\Omega / \mathrm{v}+i / 2 \bar{\ell}$ is the complex wave vector, $\mathrm{v}$ is the phase velocity, and $\bar{\ell}$ is the extinction length of the coherent intensity, arising from both elastic scattering and absorption. The extinction length $\bar{\ell}$ is related to the scattering mean free path $\ell_{s}$ and the absorption length $\ell_{a}$ by the simple expression

$$
\frac{1}{\bar{\ell}}=\frac{1}{\ell_{s}}+\frac{1}{\ell_{a}}
$$

Here $\ell_{a}$ is related to the absorption time $\tau_{a}$ by $\ell_{a}=\mathrm{v} \tau_{a}$. In the small $\omega$ limit, the frequency dependence of $\bar{\ell}$ can be neglected, so that we can take $\bar{\ell}(\Omega \pm \omega / 2) \approx \bar{\ell}(\Omega)$.

The effects of the multiple scattering are accounted for by the irreducible vertex function $U\left(\vec{r}_{1}, \vec{r}_{3} ; \vec{r}_{2}, \vec{r}_{4}\right)$. For isotropic scattering, the scattering mean free path $\ell_{s}$ is equal to the transport mean free path $\left(\ell_{s}=\ell^{*} \equiv \ell\right)$, and the lowest order contribution to the vertex function becomes [18]

$U\left(\vec{r}_{1}, \vec{r}_{3} ; \vec{r}_{2}, \vec{r}_{4}\right)=\frac{4 \pi}{\ell} \delta\left(\vec{r}_{1}-\vec{r}_{3}\right) \delta\left(\vec{r}_{1}-\vec{r}_{2}\right) \delta\left(\vec{r}_{3}-\vec{r}_{4}\right)$.

With this form for $U$, Eq. (3) generates a sum of ladder diagrams. In the absence of absorption, it can be shown that the expressions for $\langle G\rangle$ and $U$ given by Eqs. (4) and (6) satisfy the Ward identity in the $\omega=0$ limit [18]. Furthermore, when a plane wave is incident at $z=0$ on a slab containing random scatterers, $\left\langle\phi_{\Omega^{ \pm}}(\vec{r})\right\rangle$ is simply a decaying plane wave (due predominantly to scattering) with frequency $\Omega^{ \pm}$propagating in the $z$ direction (perpendicular to the slab).
Thus, after substituting Eqs. (4) and (6) into Eq. (3), the equation for $C_{\Omega}(\omega ; \vec{r})$ becomes

$$
\begin{aligned}
C_{\Omega}(\omega, \vec{r})= & \exp \left(\frac{i \omega z}{\mathrm{v}}-\frac{z}{\bar{\ell}}\right)+\frac{1}{4 \pi \ell} \int d \vec{r}_{1} \\
& \times \frac{\exp \left[\left(\frac{i \omega}{\mathrm{v}}-\frac{1}{\bar{\ell}}\right)\left(\left|\vec{r}-\vec{r}_{1}\right|\right)\right]}{\left|\vec{r}-\vec{r}_{1}\right|^{2}} \\
& \times C_{\Omega}\left(\omega, \vec{r}_{1}\right) .
\end{aligned}
$$

In Eq. (7), the fact that the source term depends only on the $z$ coordinate means $C_{\Omega}(\omega, \vec{r})$ is independent of the transverse coordinate $\vec{\rho}$. Thus $C(\omega, \vec{r})=C(\omega, z)$, and Eq. (7) becomes

$$
\begin{aligned}
C_{\Omega}(\omega, z)= & \exp \left(\frac{i \omega z}{\mathrm{v}}-\frac{z}{\bar{\ell}}\right)+\frac{1}{4 \pi \ell} \int_{0}^{L} d z_{1} H\left(z-z_{1}\right) \\
& \times C_{\Omega}\left(\omega, z_{1}\right),
\end{aligned}
$$

where

$$
H\left(z-z_{1}\right)=\int d \vec{\rho} \frac{\exp \left[\left(\frac{i \omega}{\mathrm{v}}-\frac{1}{\bar{\ell}}\right) \sqrt{\rho^{2}+\left(z-z_{1}\right)^{2}}\right]}{\rho^{2}+\left(z-z_{1}\right)^{2}} .
$$

The first term in Eq. (8) is the coherent ballistic contribution, while the second term describes the effects of scattering. This equation can be solved numerically to obtain the frequency correlation function of the transmitted waves, $C_{\Omega}(\omega, L)$, for a given set of parameters $\mathrm{v}, \ell_{,} \ell_{a}$, and $L$.

Now that we have solved for the frequency correlation function, it is straightforward to calculate the temporal variation of the transmitted intensity from Eq. (2). At the back face of the sample, Eq. (2) becomes

$$
\begin{aligned}
\left\langle|\psi(t, L)|^{2}\right\rangle= & (2 \pi)^{-2} \int d \Omega\left[\int d \omega f\left(\Omega^{+}\right) f^{*}\left(\Omega^{-}\right)\right. \\
& \left.\times C_{\Omega}(\omega, L) \exp (-i \omega t)\right] .
\end{aligned}
$$

Our experiments were performed using a Gaussian input pulse, for which $f(\Omega)$ is proportional to $\exp [-(\Omega$ $\left.-\Omega_{0}\right)^{2} / 2 \sigma^{2}$ ], where $\Omega_{0}$ is the central frequency in the pulse and $\sigma$ determines the pulse width. Then the transmitted intensity profile, normalized to the peak of the input pulse, is simply

$$
\begin{aligned}
I(t, L)= & \frac{1}{2 \pi \sigma^{2}} \int d \Omega\left[\int d \omega \exp \left\{-\left[\left(\Omega-\Omega_{0}\right)^{2}-\omega^{2} / 4\right] / \sigma^{2}\right\}\right. \\
& \left.\times C_{\Omega}(\omega, L) \exp (-i \omega t)\right] .
\end{aligned}
$$

In Eqs. (10) and (11), the limits on the integrals over frequency are from $-\infty$ to $+\infty$. Thus the fact that the correlation function obeys the symmetry relation $C_{\Omega}(-\omega, L)$ 
$=C_{\Omega}^{*}(\omega, L)$, as can been seen directly from Eqs. (8) and (9), ensures that the intensity profile $I(t, L)$ is real.

\section{EXPERIMENT}

To investigate the transition from ballistic to diffusive behavior experimentally, we use ultrasonic experiments to probe the character of acoustic pulse propagation in a strongly scattering medium consisting of 0.5 -mm-radius glass beads immersed in water. The beads have a polydispersity of about $10 \%$, making it relatively easy to pack the beads randomly, at a volume fraction of about $60 \%$, without visible indications of significant interparticle positional correlations. The thickness $L$ of the samples was varied from 1 to $10 \mathrm{~mm}$, corresponding to about $1.3-13$ mean free paths. For the thicker samples $(L>3 \mathrm{~mm})$, the beads were packed in 10-cm-diameter disk-shaped cells having thin polystyrene walls. The cells were placed in a water tank between a planewave-generating transducer and a small, 200- $\mu$ m-radius hydrophone detector [5]. The hydrophone, having transverse dimensions less than the ultrasonic wavelength, allowed the transmitted ultrasonic field to be measured in a single coherence area, or speckle spot, at the back face of the sample. This ensured that the scattered sound could be accurately measured, effectively eliminating spurious phase cancellation effects in the detector and reliably detecting the amplitude and phase of the field at a given point in the speckle pattern [5]. To collect the transmitted field in many independent speckles, the hydrophone was scanned across the sample face. For the thinnest samples $(L \leqslant 3 \mathrm{~mm})$, it was essential to eliminate the slight broadening of the detected time profiles resulting from the time taken for the scattered sound to travel obliquely through the exit wall of the sample en route to the detector. Thus the thinnest samples were placed horizontally on a low-density polyethylene surface, which has an acoustic impedance very close to that of water, and the top wall was removed, enabling the hydrophone detector to be placed within $0.5 \mathrm{~mm}$ of the sample surface. This eliminated any distortions of the signal due to the sample walls, facilitating a quantitative comparison of experiment and theory [19]. The detected wave forms were digitized and signal averaged to improve the signal-to-noise ratio and to permit subsequent analysis of the data.

We exploit the phase sensitive nature of ultrasonic transducers to separate the ballistic component from the scattered component of the transmitted acoustic fields. This ability to separate the ballistic and scattered fields is essential for a quantitative analysis of the entire transmitted wave form for thin samples, where the ballistic component, which is exponentially attenuated with thickness, can become quite large. This is illustrated in Fig. 1(a), where the total transmitted field in several different speckles is shown for a sample that is approximately two scattering mean free paths thick. At the leading edge of the transmitted signals, a coherent component, representing the ballistic pulse, is clearly seen, while at later times no correlation is evident in the amplitude and phase of the field in the different speckles. The ballistic component is extracted from the total transmitted wave form by averaging the transmitted field over many (120) speckles, as shown in Fig. 1(b); because of the random phases of the scattered signals from different speckles, the scattered wave

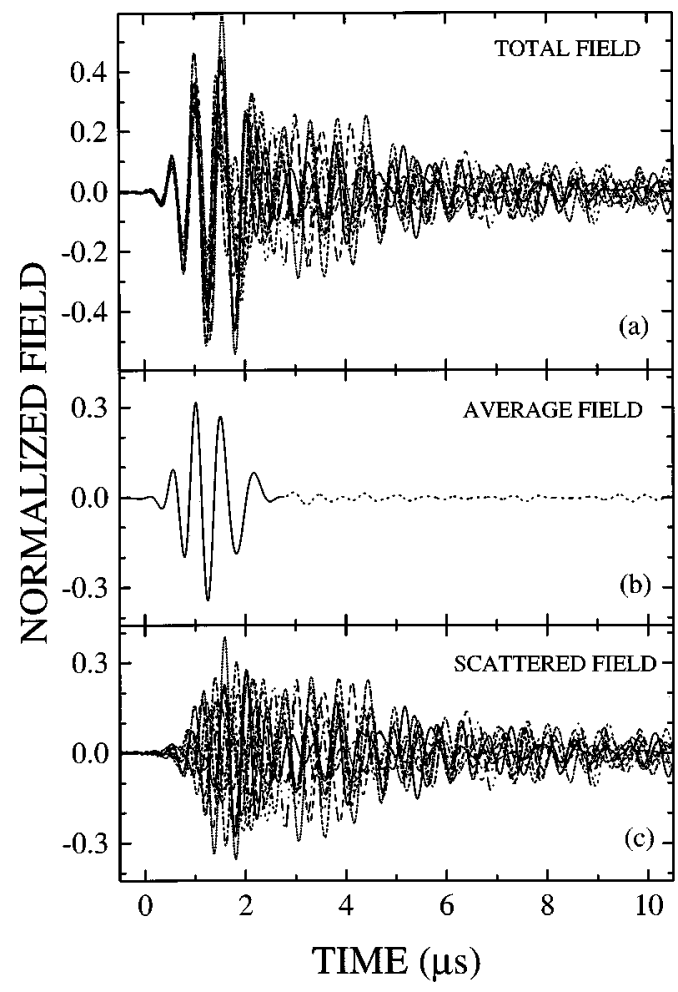

FIG. 1. (a) The total transmitted field in several independent speckles, (b) the ensemble averaged field, and (c) the scattered field for the same speckles shown in (a). The sample thickness was 1.7 $\mathrm{mm}$. The data are all normalized so that the peak amplitude of the input pulse is unity. In (b), the coherent ballistic pulse is highlighted by the solid curve.

forms cancel in the averaging, leaving only the ballistic signal, which is temporally and spatially coherent across the back face of the sample. This averaging procedure allows us to determine the true scattered wave form for each speckle by subtracting the ballistic component from the raw transmission data [see Fig. 1(c)].

We measure the frequency correlation function of the scattered acoustic fields using an approach [20] that takes advantage of the procedure outlined above for extracting the ballistic component. Since we use a short incident ultrasonic pulse, the transmitted fields contain a wide spectrum of frequencies. We can therefore determine the response function of the scattering medium directly by calculating the ratio of the complex fast Fourier transform of the scattered fields to that of the input pulse. Figure 2(a) shows the response function in a single speckle, for one of the speckles whose time profile is shown in Fig. 1. The frequency fluctuations characteristic of speckle interference are clearly seen, with the range of frequencies accessible in a single measurement extending from about 1 to over $3 \mathrm{MHz}$ in this example. These results are compared with the response function for a thicker sample, $L=5.24 \mathrm{~mm}$, in Fig. 2(b), which illustrates the expected rapid increase in fluctuation rate with sample thickness, reflecting the dominance of longer scattering paths in the thicker sample. The frequency correlation function of the scattered fields is then obtained by autocorrelating the response function and ensemble averaging over many speckles. We note that this approach measures the field correlation function directly. 


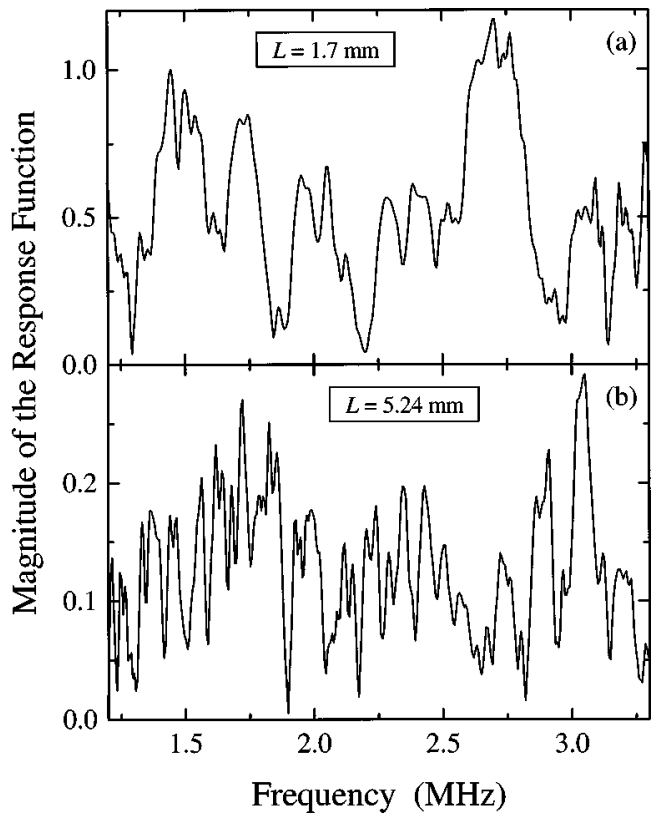

FIG. 2. The response function of the scattering medium, measured in a single coherence area, for two sample thicknesses, $L$ $=1.7 \mathrm{~mm}$ (a) and $L=5.24 \mathrm{~mm} \mathrm{(b).}$

The time dependence of the average intensity of the scattered transmitted fields is determined by squaring the envelope of the measured wave forms in each speckle, and averaging over all speckles [5]. This procedure is illustrated in Fig. 3, where in the upper panel we show the intensity profiles in several different speckles for the thicker of the two samples illustrated in Fig. 2. In the lower panel we show the ensemble-averaged intensity over all 120 speckles measured for this sample. The dashed curve in Fig. 3(b) is the intensity profile from a fit to the predictions of the diffusion approximation, as discussed below. It is worth noting that since we are able to measure the field accurately in each individual speckle, the ensemble average of the square of the field envelopes is an accurate measure of the average intensity profile. On the other hand, if each field measurement were smeared out over several speckles by using too large a detector, the result would be a mixture of field and intensity averaging; thus the intensity statistics would be seriously distorted, and a reliable measurement of the average intensity would not be possible.

\section{RESULTS AND DISCUSSION}

In Fig. 4, we compare the absolute values of the theoretical and experimental frequency correlation functions of the scattered fields $\left|C_{\Omega}^{\text {scat }}(\omega, L) / C_{\Omega}^{\text {scat }}(0, L)\right|$ for four different sample thicknesses ranging from $L=10.2$ to $1.65 \mathrm{~mm}$. The experimental frequency correlation functions were measured over a relatively narrow frequency interval centered at $\Omega / 2 \pi=2.5 \mathrm{MHz}$ to avoid possible complications due to dispersion [21,22] and scattering anisotropy [23]. For each thickness, the solid symbols represent the experimental data points, and the solid curves are the results calculated from Eq. (8) with the ballistic contribution subtracted. The solid theoretical curves are plotted for all thicknesses using the same values of $\mathrm{V}$ and $\ell$ which were determined by fitting the

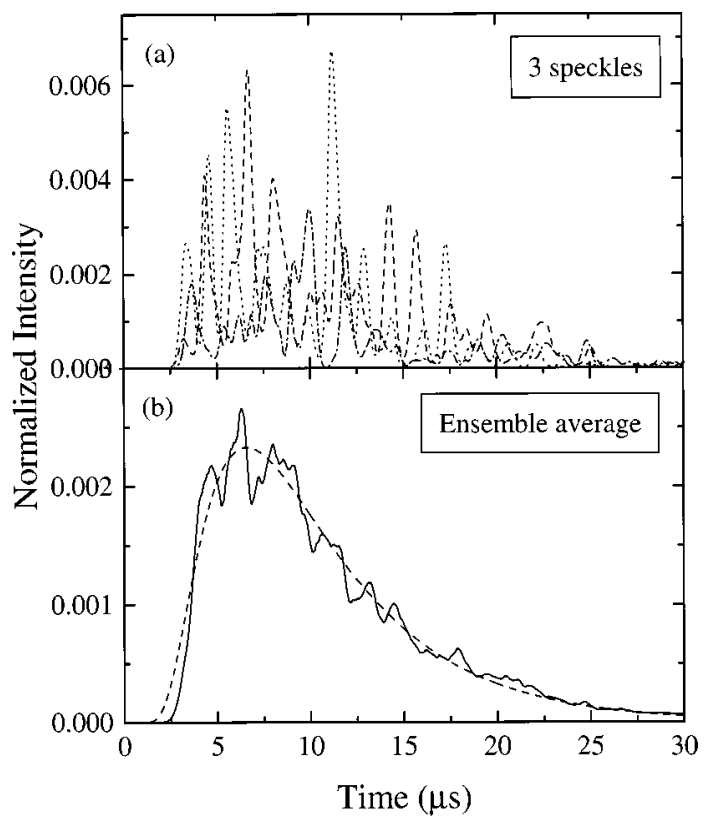

FIG. 3. (a) The square of the envelopes of the transmitted field in three different speckles for the $L=5.24 \mathrm{~mm}$ sample, and (b) theensemble averaged intensity obtained by averaging the square of the field envelopes over 120 independent speckles. The data are normalized by the peak value of the input pulse intensity. The dashed curve is a fit to the predictions of the diffusion approximation, as discussed in Sec. IV.

experimental data. Absorption is included in $\bar{l}$ using the experimentally measured value of the absorption time, $\tau_{a}$ $=\ell_{a} / \mathrm{V}=12 \mu \mathrm{s}$ [5]. Except for the thinnest sample $(L$ $=1.65 \mathrm{~mm}$ ), the agreement between the theoretical predictions and the experiment is very good, establishing the validity of our model (at least for the thicker samples) and allowing the values of the parameters $\mathrm{v}$ and $\ell$ to be reliably determined. Moreover, the best-fitted values are $\mathrm{V}$ $=1.3 \mathrm{~mm} / \mu \mathrm{s}$ and $\ell=0.76 \mathrm{~mm}$, which agree very well with the experimental values of $1.33 \pm 0.03 \mathrm{~mm} / \mu \mathrm{s}$ for the phase velocity $\mathrm{v}_{p}$, and $0.75 \pm 0.1 \mathrm{~mm}$ for the scattering mean free path [5]. The dashed curves for each thickness are the results

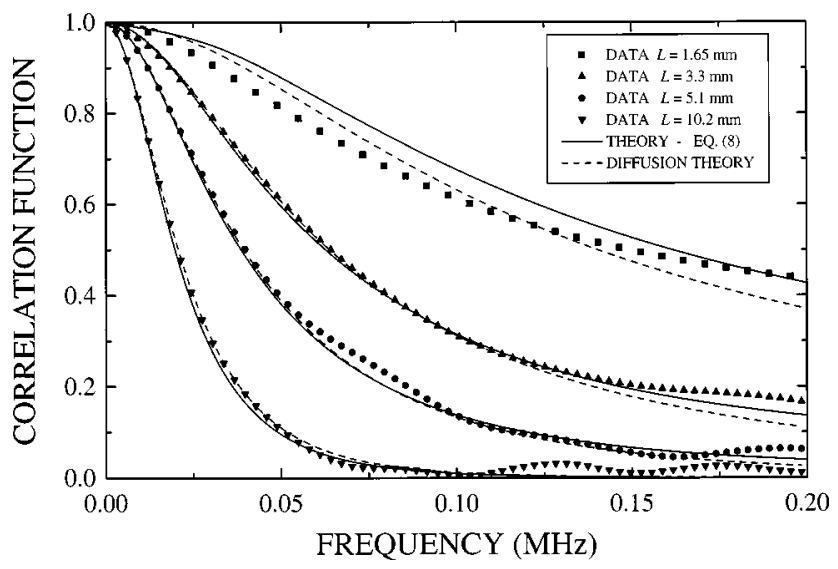

FIG. 4. The absolute value of the frequency correlation function for four sample thicknesses. The solid symbols represent the experimental data. The solid curves are calculated from Eq. (8), while the dashed curves are the solutions to the diffusion equation. 
obtained from the solution of the diffusion equation with the correct boundary conditions for our samples using the same value of the diffusion coefficient, $D=0.43 \mathrm{~mm}^{2} / \mu \mathrm{s}$, as was found previously in thick samples by fitting the time profile of the average transmitted intensity [5]. For the three thicker samples, where the ratio $L / /$ ranges from 13 to 4.3 , the predictions of the diffusion approximation agree well with our theoretical and experimental results, demonstrating that the propagation of the scattered waves is diffusive for sample thicknesses down to about four mean free paths.

While the diffusion approximation is adequate to describe the transport when $L / \ell \geqslant 4$, it must ultimately fail for even thinner samples. This breakdown of the diffusion approximation is clearly seen in our results for the frequency correlation function of the thinnest sample shown in Fig. 4, where $L=1.65 \mathrm{~mm}(L / \ell \cong 2.2)$. For this thickness, the predictions of the diffusion approximation do not agree with either the experimental data or our theoretical model. Figure 4 shows that the experimental correlation function falls off more rapidly with frequency than the theoretical predictions in the low frequency region. In the time domain, this narrowing of the frequency correlation function implies a slower decay of the transmitted intensity in the long-time limit. This is similar to a recent observation in time-resolved optical experiments [10], where it was found for thin samples that the diffusion coefficient obtained by fitting the long-time exponential decay of the transmitted intensity is progressively reduced as the sample thickness is decreased; in these optical experiments, however, this slow decay of the intensity at long times was observed for sample thicknesses up to $L / \ell^{*} \approx 8$, which is a larger crossover value than we find in our system. The narrowing of the frequency correlation function in our thinnest sample may arise from the interference of multiply scattered waves, which is ignored in diffusion theory and has not been included in the solution of the Bethe-Salpeter equation studied here. When the sample thickness becomes comparable to the transport mean free path, the long-time behavior of the transmitted intensity and the corresponding small-frequency behavior of the correlation function are due to waves that have undergone many multiple scatterings in lateral directions inside the sample. As a result of the reduced effective dimensionality for propagation in the plane of the sample, wave interference is likely to become more important.

To investigate the transport behavior of thin samples further, we focus on measurements of the time-resolved transmitted intensity; this has the important advantage of allowing us to see directly how the transport depends on path length. Figure 5 shows our data for both the scattered intensity (open symbols) and the total transmitted intensity (solid symbols); the difference gives the ballistic component, which is a relatively small contribution for the $3.3-\mathrm{mm}$ sample, but which dominates the early time behavior of the transmitted intensity for the thinner $1.65-\mathrm{mm}$ sample. In Fig. 5, all the data are normalized to the peak intensity at $t=0$ of the input pulse. We calculate the normalized transmitted intensity theoretically using Eq. (11). Because of the wide range of frequencies contained in the input pulse spectrum, it is necessary to account for the effects of dispersion, which we include by solving Eq. (11) numerically for $I(t, L)$ at several different frequencies $\Omega / 2 \pi$ within the experimental pulse

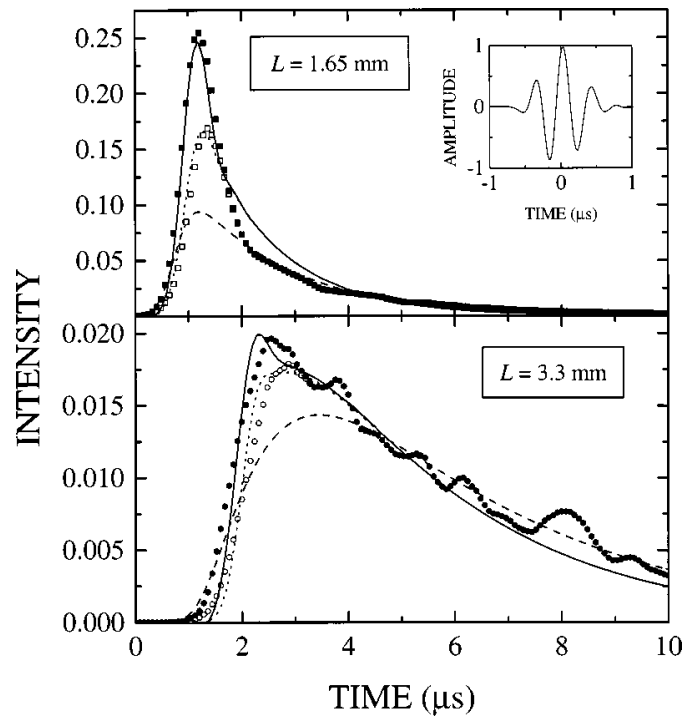

FIG. 5. The time profile of the transmitted intensity, normalized so that the peak of the input pulse intensity is 1 , for two thin samples. The solid symbols and curves are experimental and theoretical results for the total transmitted intensity, while the open symbols and dotted curves represent the scattered contributions only. These theoretical curves are calculated using the model developed in this paper, while the predictions of the diffusion model are represented by the dashed curves. The inset shows the time profile of the input pulse field.

spectrum, from 1.5 to $3.5 \mathrm{MHz}$, using the experimental values of $\mathrm{v}_{p}, \ell_{s}$, and $\tau_{a}$ over this frequency range $[21,24]$. This gives the scattered and total intensity time profiles shown by the dotted and solid curves in Fig. 5. Note that here we have plotted the data and theory using a linear scale for $I(t, L)$ in order to show most clearly the behavior at early times where deviations from diffusive propagation are expected to be most extreme; thus the deviations in the longtime tail noted above for the thinner sample are not very apparent in this figure. The agreement between theory and experiment for the early time behavior is quite satisfactory, although, for the thinnest sample, somewhat poorer agreement is also found for the shape of the scattered pulse profile at early times, where the effects of anisotropic scattering, local microstructure variations, and boundary conditions become more important and complicate a detailed analysis of the pulse shape. For comparison, in Fig. 5 we also show the predictions of the diffusion approximation for these samples. Not surprisingly, the diffusion approximation completely fails to predict the observed behavior for the thinner sample at times less than about $2 \mu \mathrm{s}$, which corresponds to a path length of about four scattering mean free paths. For the 3.3-mm sample, where the long-time tail of the transmitted intensity is very well described by the diffusion approximation (cf. Fig. 4), deviations in the shape of the diffuse intensity profile are beginning to show up at early times, where the data and predictions of our Bethe-Salpeter model exhibit a somewhat sharper peak.

To elucidate the character of wave transport in thin samples, we focus on the peak arrival time of the scattered component of transmitted intensity, since the location of the peak in the scattered intensity time profile is relatively insensitive to sample-specific details, providing a robust measure 


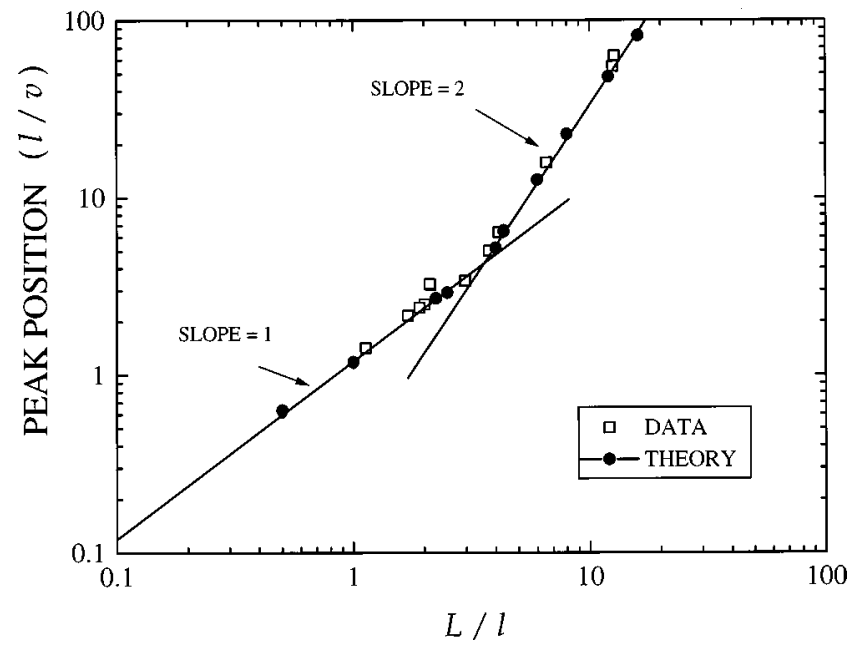

FIG. 6. The peak position in the time profile of the transmitted intensity plotted as a function of sample thickness $L / \ell$.

of the general characteristics of wave transport. In Fig. 6, we show a log-log plot of the peak positions (in normalized units of $\ell / \mathrm{v}_{g}$, where $\mathrm{v}_{g}$ is the group velocity) as a function of $L / \ell$. For the experimental data, the effects of absorption have been removed by dividing the time profiles by the measured exponential absorption factor $e^{-t / \tau_{a}}$. To make a meaningful comparison between theory and experiment, the theoretical curves are calculated for the same input pulse shape as was used in the experiments. Remarkably, we see that both the theoretical and experimental points clearly follow a twosegment curve, indicated by the two solid lines. The lower segment, with $L / \ell<3$, has a slope of 1 characteristic of ballistic propagation, whereas the upper segment, with $L / \ell$ $>3$, has a slope of 2 characteristic of diffusive behavior [25]. These results demonstrate that even when the ballistic component is subtracted off, the scattered component still behaves ballistically for thin samples at early times, and displays a surprisingly sharp transition from ballistic to diffusive behavior at $L / \ell \approx 3$.

The most remarkable feature of these results is that ballistic characteristics prevail in the early time behavior of the scattered waves for sample thicknesses up to and including this crossover thickness. The origin of this behavior lies in the dominance of single scattering at the transmitted peak, a condition which appears to persist as long as the sample thickness is less than about three mean free paths. Since the most probable path for single scattering is indistinguishable from the ballistic path (because the singly scattered intensity is attenuated exponentially with path length), these scattered signals take on a ballistic character. Furthermore, even though the arrival times of the scattered "ballistic" peaks are delayed by about $20 \%$ with respect to the ballistic pulse, the ballistic character of the scattered waves is not destroyed, because this shift results from the convolution of the asymmetric scattered time profile with the input pulse.

We have also investigated the question of whether scattering anisotropy has an important effect on the ballistic to diffusion transition shown in Fig. 6. Under these circumstances, the transport mean free path $\ell^{*}$ becomes greater than the scattering mean free path $\ell_{s}$, and the solution of the Bethe-Salpeter equation becomes more difficult. We have therefore studied the crossover behavior in this case using
Monte Carlo simulations. For a $\delta$-function input pulse, the time-resolved transmitted intensity is obtained from the statistical distribution of the number of times a pulse reaches the back surface between times $t$ and $t+d t$, having performed a random walk through the sample. For each step of the random walk, a travel distance $\ell$ is determined from a random number generated from the exponential distribution $P_{s c}(\ell)=\ell_{s}^{-1} \exp \left(-\ell \mid \ell_{s}\right)$. The corresponding travel time $t$ is $\ell / \mathrm{V}$. The direction of each step is also random and is generated according to the Henyey-Greenstein distribution function $P(\cos \theta, \phi)=(1 / 4 \pi)\left(1-g^{2}\right) /\left(1+g^{2}-2 g \cos \theta\right)^{3 / 2}$ [26]. Here $g$ is the anisotropy factor, describing the average of $\cos \theta$, i.e.,

$$
g=\langle\cos \theta\rangle=\int_{-1}^{1} d(\cos \theta) \int_{0}^{2 \pi} d \phi P(\cos \theta, \phi) \cos \theta .
$$

Thus $\ell^{*}=\ell_{s} /(1-g)$, and $g$ goes from 0 in the isotropic limit to 1 in the anisotropic limit. By plotting the peak arrival time of the scattered component as a function of $L / \ell^{*}$ at two different degrees of anisotropy, $g=0.5$ and 0.8 , we found that the transition from ballistic to diffusive behavior occurs when $L$ is between $3 \ell^{*}$ and $4 \ell^{*}$. Thus the sample thickness at which the crossover occurs is essentially unaffected by anisotropy, providing the thickness is measured in units of the transport mean free path. However, the crossover becomes less sharp when the scattering is more anisotropic.

It should be pointed out that the crossover thickness we have found here is not universal, as this thickness depends on the physical quantity under study as well as the sourcedetector geometry. Examples illustrating the range of crossover thicknesses that have been observed previously $[4,9-$ 12] are discussed in Sec. I. To investigate the possible effects of source geometry on the ballistic to diffusive transition, we have also performed experiments using a focused source, where the incident beam was only about two wavelengths wide, and thus was a good approximation to a point source. In this case, we find that the crossover thickness determined from the peak arrival time is found to increase to $L / \ell^{*} \approx 5$, about $50 \%$ larger than for an extended beam. A similar trend is seen in the thickness dependence of the overall pulse shape and frequency correlation function, showing again how experimental conditions influence the sample thickness at which the crossover from ballistic to diffusive behavior becomes manifest. Indeed, the fact that the crossover thickness depends on the quantity being investigated, while having obvious consequences for correctly interpreting experimental results on thin samples, also reflects the value of using a variety of approaches to probe the underlying changes in wave character at the transition, since different approaches will be sensitive to different aspects of these more universal features.

\section{CONCLUSIONS}

Our work integrates theoretical calculations with experiments to yield insights into the ballistic to diffusive transition in thin samples of strongly scattering materials. Our theory and experiments provide information about the minimum sample thickness for diffusive behavior to occur; our results pertain to slab-shaped samples illuminated by extended pulsed sources, an important situation not only in ultrasonic 
applications such as diffusing acoustic wave spectroscopy [27] but also in optics, where pulsed sources are becoming more frequently used to probe strongly scattering biological tissues. Furthermore, our results identify a ballistic regime of scattered signals that may be most useful for time-gated imaging techniques in strongly scattering materials. In addition, we find that wave transport at the dominant path length in pulsed experiments undergoes a sharp transition as a function of sample thickness from behavior dominated by single scattering to diffusive behavior, so that it may not always be necessary to consider a complicated intermediate regime that separates ballistic and diffusive propagation. We emphasize that although we have concentrated on acoustic waves in this work, our theoretical approach, based on the Bethe-Salpeter equation, is quite general and can be applied to any wave satisfying the classical wave equation; thus our main conclusions should apply to all classical waves, and therefore should be valid for electromagnetic waves as well.

\section{ACKNOWLEDGMENTS}

We thank K. S. Beaty for her assistance with some of the experiments, Tse Man for his assistance with the Monte Carlo simulations, and the NSERC of Canada, NATO, the Hong Kong RGC (Grant No. HKUST 6137/97P), and the NSF (Grant DMR96-31279) for their support of this project.
[1] P. Sheng, Introduction to Wave Scattering, Localization, and Mesoscopic Phenomena (Academic, San Diego, 1995).

[2] M. P. van Albada and A. Lagendijk, Phys. Rev. Lett. 55, 2692 (1985).

[3] P. E. Wolf and G. Maret, Phys. Rev. Lett. 55, 2696 (1985).

[4] J. H. Li, A. A. Lisyansky, T. D. Cheung, D. Livdan, and A. Z. Genack, Europhys. Lett. 22, 675 (1993).

[5] J. H. Page, H. P. Schriemer, A. E. Bailey, and D. A. Weitz, Phys. Rev. E 52, 3106 (1995).

[6] J. F. de Boer, M. P. van Albada, and A. Lagendijk, Phys. Rev. B 45, 658 (1992).

[7] J. M. Drake and A. Z. Genack, Phys. Rev. Lett. 63, 259 (1989).

[8] D. J. Pine, D. A. Weitz, P. M. Chaikin, and E. Herbolzheimer, Phys. Rev. Lett. 60, 1134 (1988).

[9] D. A. Weitz, D. J. Pine, P. N. Pusey, and R. J. A. Tough, Phys. Rev. Lett. 63, 1747 (1989).

[10] R. H. J. Kop, P. de Vries, R. Sprik, and A. Lagendijk, Phys. Rev. Lett. 79, 4369 (1997).

[11] K. M. Yoo, F. Liu, and R. R. Alfano, Phys. Rev. Lett. 64, 2647 (1990).

[12] K. M. Yoo and R. R. Alfano, Opt. Lett. 15, 320 (1990).

[13] F. C. MacKintosh and S. John, Phys. Rev. B 40, 2383 (1989).

[14] P.-A. Lemieux, M. U. Vera, and D. J. Durian, Phys. Rev. E 57, 4498 (1998).

[15] A. A. Chabanov and A. Z. Genack, Phys. Rev. E 56, R1338 (1997).

[16] Photon Migration and Imaging in Random Media and Tissues, edited by B. Chance and R. R. Alfano [Proc. SPIE, Vol. 1888 (1993)].
[17] Z.-Q. Zhang and P. Sheng, in Photonic Band Gap Materials, edited by C. M. Soukoulis (Kluwer, Dordrecht, 1996), p. 715.

[18] M. B. van der Mark, M. P. van Albada, and A. Lagendijk, Phys. Rev. B 37, 3575 (1988).

[19] Note that the solution of the Bethe-Salpeter equation includes the effect of the interface between water and the sample, so that the boundary conditions implicit in our model are in excellent correspondence with this experimental geometry. Also, even when the polystyrene walls were used, their effect on generating additional internal reflections should very small, as the impedance of polystyrene and water are also quite similar.

[20] I. P. Jones, J. H. Page, and D. A. Weitz (unpublished).

[21] J. H. Page, P. Sheng, H. P. Schriemer, I. Jones, X. Jing, and D. A. Weitz, Science 271, 634 (1996).

[22] H. P. Schriemer, M. L. Cowan, J. H. Page, P. Sheng, Z. Liu, and D. A. Weitz, Phys. Rev. Lett. 79, 3166 (1997).

[23] Note that, at this frequency, we have shown previously (Ref. [5]) that $\ell^{*}$ and $l_{s}$ are essentially the same, their values being indistinguishable within experimental error. Thus it is valid to interpret these data using our isotropic scattering model.

[24] J. H. Page, H. P. Schriemer, I. P. Jones, P. Sheng, and D. A. Weitz, Physica A 214, 64 (1997).

[25] The robustness of the theoretical predictions shown in Fig. 3 is illustrated by the fact that identical results are obtained for the dispersionless case, where $\mathrm{v}=\mathrm{v}_{p}$ is independent of frequency, provided that the peak positions are calculated in units of $\ell / \mathrm{v}_{p}$.

[26] L. G. Henyey and J. L. Greenstein, Astrophys. J. 93, 70 (1941).

[27] M. L. Cowan, J. H. Page, and D. A. Weitz (unpublished). 